\title{
THE MASSIVE DARK CORONA OF OUR GALAXY
}

\author{
K.C. FREEMAN \\ Mount Stromlo and Siding Spring Observatories \\ The Australian National University \\ Canberra, AUSTRALIA
}

\begin{abstract}
From their rotation curves, most spiral galaxies appear to have massive dark coronas. The inferred masses of these dark coronas are typically 5 to 10 times the mass of the underlying stellar component. I will review the evidence that our Galaxy also has a dark corona. Our position in the galactic disk makes it difficult to measure the galactic rotation curve beyond about $20 \mathrm{kpc}$ from the galactic center. However it does allow several other indicators of the total galactic mass out to very large distances. It seems clear that the Galaxy does indeed have a massive dark corona. The data indicate that the enclosed mass within radius $R$ increases like $M(R) \approx R(\mathrm{kpc}) \times 10^{10} M_{\odot}$, out to a radius of more than $100 \mathrm{kpc}$. The total galactic mass is at least $12 \times 10^{11} M_{\odot}$.
\end{abstract}

\section{Summary}

A full version of this paper will appear elsewhere (Freeman 1995). Here I present the main conclusions from the individual indicators of the galactic mass distribution.

- The mass of the known luminous components of the Galaxy is in the range ( 5 to 12$) \times 10^{10} M_{\odot}$.

- From the rotation curve of the Galaxy, $M(20 \mathrm{kpc}) \approx 22 \times 10^{10} M_{\odot}$, which is already at least double the estimated mass of the visible components.

- The escape velocity at the solar radius, estimated from high velocity stars in the solar neighborhood, indicates that the galactic mass > $30 \times 10^{10} M_{\odot}$. 
- From the kinematics of distant stars and satellites, $M(50 \mathrm{kpc}) \approx 40 \times$ $10^{10} M_{\odot}$.

- The timing arguments from the radial velocities and distances of M31 and Leo I give a consistent asymptotic mass estimate $M_{\text {total }}$ of at least $120 \times 10^{10} M_{\odot}$.

\section{Conclusion}

The data are consistent with a mass distribution $M(R) \approx R(\mathrm{kpc}) \times 10^{10}$ $M_{\odot}$ (corresponding to a flat rotation curve with $V_{c} \approx 220 \mathrm{~km} \mathrm{~s}^{-1}$ ), extending out to $R \geq 100 \mathrm{kpc}$. The inferred ratio of the mass of the dark corona to the mass of the visible components of the Galaxy is at least 10 .

Recently Kochanek (1995) used a Jaffe (1983) model to represent the mass distribution of the Galaxy, and estimated the parameters for this model from the mass indicators taken together. He concludes that the mass within $50 \mathrm{kpc}$ is $(54 \pm 13) \times 10^{10} M_{\odot}$, which agrees well with the run of $M(R)$ given here from the mass indicators taken individually.

\section{References}

Freeman, K.C., 1995, in "Unsolved Problems of the Milky Way" (IAU Symposium 169), ed L. Blitz (Dordrecht: Kluwer), in press

Jaffe, W., 1983, MNRAS, 202, 995

Kochanek, C.S., 1995, ApJ, in press 